\title{
Is it all in the Wrist? The Potential of Activity Trackers to Rise Physical Activity in Cancer Survivors
}

ISSN: 2637-773X

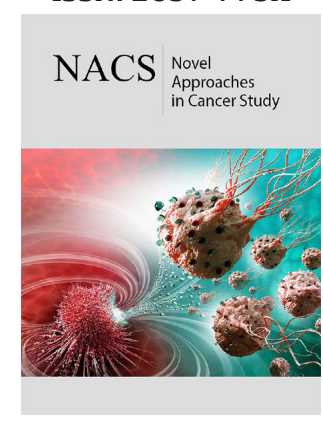

*Corresponding author: Luis Felipe Ribeiro Pinto, Molecular Carcinogenesis Program, Instituto Nacional de Câncer, Rio de Janeiro-RJ, Brasil

Submission: 㘹 March 29, 2019

Published: :-1-ill April 23, 2019

Volume 2 - Issue 4

How to cite this article: Fernando Tadeu Frajacomo, Mariana Severo Ramundo, Sheila Coelho Soares-Lima, Flavia Nascimento de Carvalho, Pedro Nicolau-Neto, Nathalia Meireles Da Costa, Luis Felipe Ribeiro Pinto*. Is it all in the Wrist? The Potential of Activity Trackers to Rise Physical Activity in Cancer Survivors. Nov Appro in Can Study. 2(4) NACS.000542.2019.

DOI: $10.31031 /$ NACS.2019.02.000542

Copyright@ Luis Felipe Ribeiro Pinto, This article is distributed under the terms of the Creative Commons Attribution 4.0 International License, which permits unrestricted use and redistribution provided that the original author and source are credited.

\author{
Fernando Tadeu Frajacomo, Mariana Severo Ramundo, Sheila Coelho \\ Soares-Lima, Flavia Nascimento de Carvalho, Pedro Nicolau-Neto, Nathalia \\ Meireles Da Costa, Luis Felipe Ribeiro Pinto* \\ Molecular Carcinogenesis Program, Instituto Nacional de Câncer, Brasil
}

\begin{abstract}
A growing body of evidence suggests that higher levels of physical activity are inversely associated with treatment side-effects and mortality in cancer survivors. Conversely, a limited percentage of cancer patients achieve physical activity goals and fitness compared with age-matched peers or other chronic medical conditions. In the last years, the next generation of activity trackers has integrated several parameters of physical fitness and mobility measurements. These multi-task devices go beyond objective physical activity measurements. Due to its functioning based on integrated platforms, clinicians may experience a unique opportunity to incorporate more active behaviors into their patients' lives. This mini-review will discuss the advantages, challenges and future directions of most recent activity trackers in oncology.
\end{abstract}

Keywords: Wearable technology; Cancer survivors; Physical activity; Fitness

\section{Introduction}

To date, higher levels of physical activity are associated with benefits across the natural history of cancer, from primary prevention to survivorship [1]. Encouraging results have also been acquired in the response to treatment and showed that physical activity favored better clinical outcomes and expanded survival rates in a variety of cancer types [2]. Despite the evidence of physical activity benefits, only a low percentage of cancer patients are achieving desirable activity goals. The latest multi-sensor activity trackers allow users and clinicians to assess broad functional metrics including step counting, heart frequency, sedentary behavior, sleep patterns and metabolic consumption, depending on the device model and the scientific purpose [3]. Since activity trackers are gaining recognition across health conditions, including cancer, it is worth to further explore the possibilities and limitations of such approach. Activity trackers have gained focus in clinical oncology since regular self-reported questionnaires are prone not only to bias, but also poorly correlated with physical function [4]. In addition, current physical activity programs have expanded beyond the clinical setting, requiring more independence and information to cancer survivors and clinicians [5]. As a result, a more objective, standardized, and accessible approach to implementing and monitoring physical activity programs in clinical oncology is warranted and may represent a great innovation on cancer treatment. This mini-review aims to address the role of activity trackers as a supportive strategy on two relevant endpoints in the oncology field: fitness gains and physical activity behaviors.

The opportunity of real-time monitoring and remotely obtaining feedback from cancer patientsthroughoutanunsupervisedinterventiondeservesnotice.Trialscomparing supervised versus unsupervised training programs tend to report superior fitness achievements in the supervised arm [6,7]. However, the increment of activity trackers to unsupervised exercised sessions may approximate fitness achievements using either strategy. One recent randomized trial study focusing on breast cancer survivors indicated that using wearable activity trackers along 12 weeks increased both total physical activity (PA) and $\mathrm{VO}_{2 \max }$ (defined as the highest rate of oxygen consumption during exercise, considered the gold standard parameter of cardiorespiratory fitness), following unsupervised intervention [8]. Intriguingly, $\mathrm{VO}_{2}$ (defined 
as the rate of oxygen consumption during exercise) improvements ( $\sim 20 \%$ in 12 weeks) reported by authors were comparable to two other trials using traditionally supervised, moderate or highintensity exercise, in cancer survivors $[9,10]$. However, a pilot study focusing on 19 childhood cancer survivors using activity trackers for six months showed mild improvements in vigorous $\mathrm{PA}$ and $\mathrm{VO}_{2 \max }$ [11]. Yet, different protocols of fitness assessment, age, and distinct baseline characteristics across studies turn these comparisons more difficult. Thus, it is uncertain if wearing multisensor trackers combined with unsupervised exercise sessions will be critical to achieving higher fitness, although it is currently a promising tool to be considered.

Another relevant feature of activity tracker is its potential to promote PA behavior and precisely quantify it. PA is defined as any body movement that requires energy consumption further than resting. Recognized cancer agencies advocate 150-min/week of moderate to vigorous intensity of leisure-time PA as the minimum amount for an individual to be considered "active" [3,12]. However, it should be interpreted as a category beyond regular daily movements. Population-based studies in cancer using objective trackers (i.e accelerometers) mostly report a higher amount of "lowactivity" patients than regular questionnaire-based studies [13,14]. One reason for these discrepant results could be the fact that accelerometer-derived studies are capable of objectively stratifying physical activity according to intensity categories (light, moderate or vigorous) [15]. Since novel sophisticated activity trackers capture all physical movements leading to total accumulated PA time much broader than the 150-min/week, these thresholds should be reconsidered to avoid an apparent misconception of reality. In the study of McNeil and colleagues (2019), the eligibility criteria included low activity participants $(<60$-min/week of vigorous activity), whereas baseline moderate to vigorous PA amount was 92 $\mathrm{min} /$ day on average when measured by newer generation trackers [8]. Addressing this question, Thompson et al. [16] recommended that PA classifications should be calibrated. As such, the authors proposed that moderate to vigorous PA amount should be 5 to 7-fold greater than the traditional 150-min/week when measured by novel activity trackers. In the near future, the next generation activity trackers will be able to collect and cross information about heart frequency, duration of the activity, movement pattern and metabolic consumption, thus being capable of discriminating regular daily movements from leisure-time activities.

There are further advantages of combining activity trackers to home-based/unsupervised exercise programs, and some trials were launched to test their efficacy in endometrial [17] and childhood [18] cancer survivors. In addition to more accurate, objective, and specified PA assessments, activity trackers used along cancer care continuum can act as an interface between patients and clinicians to facilitate rapid feedback, stimulate higher adherence to either exercise programs or therapeutic regimes. This is particularly relevant for elderly cancer patients to monitor the adverse effects of the polypharmacy approach [19]. Prior to a broader implementation of activity trackers use in oncology, some limitations should be addressed. Age, previous PA behavior and general health conditions may impact on adherence. For instance, tumors that induce a great tumor burden such as lung, pancreas, esophagus or neurological tumors, and therefore prone to lower patient mobility, may need special attention. Finally, the costs of wireless monitoring behavior go beyond purchasing the device, requiring an oncology team specifically trained for getting the most out of these devices and tailoring exercise prescription.

While a more patient-centered therapy is desirable in the oncology setting, activity tracker studies may help filling this gap. Recentadvancements in fitness technologies have provided qualified exercise professionals with new opportunities to incorporate more active behaviors into their patients' lives. Whether activity trackers will facilitate delivering exercise programs to patients with cancer is still uncertain. Surely, more studies are needed to replicate findings and extend the body of evidence. Still, the role of a multiprofessional team is and will be crucial in cancer surveillance. However, a novel and promising allied may be on our wrists.

\section{Conflict of Interest}

The authors declare no conflict of interest.

\section{References}

1. Courneya KS, Friedenreich CM (2007) Physical activity and cancer control. Semin Oncol Nurs 23(4): 242-252.

2. Phillips SM, Collins LM, Penedo FJ, Courneya KS, Welch W, et al. (2018) Optimization of a technology-supported physical activity intervention for breast cancer survivors: Fit2Thrive study protocol. Contemporary clinical trials 3(66): 9-19.

3. Cormie P, Atkinson M, Bucci L, Cust A, Eakin E, et al. (2018) Clinical oncology society of Australia position statement on exercise in cancer care. The Medical Journal of Australia 209(4):184-187.

4. Mafra Cabral A, Pinheiro MM, Castro CHM, De Mello MT, Tufik S, et al. (2018) Physical activity questionnaires do not accurately estimate fitness in older women. Journal of aging and physical activity 26(1): 1-6.

5. Schmidt MLK, Ostergren P, Cormie P, Ragle AM, Sonksen J, et al. (2019) "Kicked out into the real world": Prostate cancer patients' experiences with transitioning from hospital-based supervised exercise to unsupervised exercise in the community. Supportive Care Cancer 27(1): 199208.

6. Sweegers MG, Altenburg TM, Chinapaw MJ, Kalter J, Verdonck-de Leeuw IM, et al. (2018) Which exercise prescriptions improve quality of life and physical function in patients with cancer during and following treatment? A systematic review and meta-analysis of randomised controlled trials. British journal of sports medicine 52(8): 505-513.

7. Westphal T, Rinnerthaler G, Gampenrieder SP, Niebauer J, Thaler J, et al. (2018) Supervised versus autonomous exercise training in breast cancer patients: A multicenter randomized clinical trial. Cancer Medicine 7(12): 5962-5972.

8. McNeil J, Brenner DR, Stone CR, O’Reilly R, Ruan Y, et al. (2019) Activity tracker to prescribe various exercise intensities in breast cancer survivors. Medicine and Science in Sports and Exercise 51(5): 930-940.

9. Kampshoff CS, Chinapaw MJ, Brug J, Twisk JW, Schep G, Nijziel MR, et al. (2015) Randomized controlled trial of the effects of high intensity and low-to-moderate intensity exercise on physical fitness and fatigue in cancer survivors: Results of the resistance and endurance exercise after chemotherapy (REACT) study. BMC medicine 29(13): 275.

10. Martin EA, Battaglini CL, Hands B, Naumann F (2015) Higher-intensity exercise results in more sustainable improvements for $\mathrm{VO}_{2}$ peak for breast and prostate cancer survivors. Oncology nursing forum 42(3): 241-249. 
11. Le A, Mitchell HR, Zheng DJ, Rotatori J, Fahey JT, et al. (2017) A homebased physical activity intervention using activity trackers in survivors of childhood cancer: A pilot study. Pediatr Blood Cancer 64(2): 387-394.

12. Schmitz KH, Courneya KS, Matthews C, Demark-Wahnefried W, Galvao DA, et al. (2010) American college of sports medicine roundtable on exercise guidelines for cancer survivors. Medicine and Science in Sports and Exercise 42(7): 1409-1426.

13. Smith WA, Nolan VG, Robison LL, Hudson MM, Ness KK (2011) Physical activity among cancer survivors and those with no history of cancera report from the National Health and Nutrition Examination Survey 2003-2006. American Journal of Translational Research 3(4): 342-350.

14. Borowski TKM, Gennuso KP, Bertram L (2017) Accelerometer-derived physical activity and sedentary time by cancer type in the United States. PloS One 12(8): 1-12.

15. Pedisic Z, Bauman A (2015) Accelerometer-based measures in physical activity surveillance: Current practices and issues. British journal of sports medicine 49(4): 219-223.
16. Thompson D, Batterham AM, Peacock OJ, Western MJ, Booso R (2016) Feedback from physical activity monitors is not compatible with current recommendations: A recalibration study. Preventive Medicine 91: 389394.

17. Cattivelli R, Castelnuovo G, Musetti A, Varallo G, Spatola CAM, et al. (2018) ACT on HEALTH study protocol: Promoting psychological flexibility with activity tracker and mHealth tools to foster healthful lifestyle for obesity and other chronic health conditions. Trials 19(1): 659.

18. Mendoza JA, Baker KS, Moreno MA, Whitlock K, Lambertz MA, et al. (2017) A fitbit and facebook mHealth intervention for promoting physical activity among adolescent and young adult childhood cancer survivors: A pilot study. Pediatric Blood \& Cancer 64(12).

19. Balducci L, Goetz-Parten D, Steinman MA (2013) Polypharmacy and the management of the older cancer patient. Annals of Oncology 24(7): vii36-vii40.

For possible submissions Click below: 